\title{
ALL TIGHT DESCRIPTIONS OF 3-STARS IN 3-POLYTOPES WITH GIRTH 5
}

\author{
Oleg V. Borodin ${ }^{1}$ \\ Institute of Mathematics Siberian Branch \\ Russian Academy of Sciences \\ Novosibirsk, 630090, Russia \\ e-mail: brdnoleg@math.nsc.ru \\ AND \\ Anna O. Ivanova ${ }^{2}$ \\ Ammosov North-Eastern Federal University \\ Yakutsk, 677000, Russia \\ e-mail: shmgnanna@mail.ru
}

\begin{abstract}
Lebesgue (1940) proved that every 3-polytope $P_{5}$ of girth 5 has a path of three vertices of degree 3 . Madaras (2004) refined this by showing that every $P_{5}$ has a 3-vertex with two 3-neighbors and the third neighbor of degree at most 4. This description of 3 -stars in $P_{5} \mathrm{~s}$ is tight in the sense that no its parameter can be strengthened due to the dodecahedron combined with the existence of a $P_{5}$ in which every 3 -vertex has a 4-neighbor.

We give another tight description of 3 -stars in $P_{5}$ s: there is a vertex of degree at most 4 having three 3 -neighbors. Furthermore, we show that there are only these two tight descriptions of 3-stars in $P_{5} \mathrm{~s}$.

Also, we give a tight description of stars with at least three rays in $P_{5} \mathrm{~s}$ and pose a problem of describing all such descriptions. Finally, we prove a structural theorem about $P_{5}$ s that might be useful in further research.
\end{abstract}

Keywords: 3-polytope, planar graph, structure properties, $k$-star.

2010 Mathematics Subject Classification: 05C15.

\footnotetext{
${ }^{1}$ This work was supported by grants 16-01-00499 and 15-01-05867 of the Russian Foundation for Basic Research and President Grant for Government Support of the Leading Scientific Schools of the Russian Federation NSh-1939.2014.1.

${ }^{2}$ The author's work was performed as a part of government work "Organizing research" and supported by grant 15-01-05867 of the Russian Foundation for Basic Research.
} 


\section{REFERENCES}

[1] O.V. Borodin, Solution of Kotzig's and Grünbaum's problems on the separability of a cycle in a planar graph, Mat. Zametki 46 (1989) 9-12, in Russian.

[2] O.V. Borodin, Structural properties of planar maps with the minimal degree 5, Math. Nachr. 158 (1992) 109-117. doi:10.1002/mana.19921580108

[3] O.V. Borodin and D. Sanders, On light edges and triangles in planar graphs of minimal degree five, Math. Nachr. 170 (1994) 19-24. doi:10.1002/mana.19941700103

[4] O.V. Borodin and D.R. Woodall, Short cycles of low weight in normal plane maps with minimum degree 5, Discuss. Math. Graph Theory 18 (1998) 159-164. doi:0.7151/dmgt.1071

[5] O.V. Borodin and A.O. Ivanova, Describing 4-stars at 5-vertices in normal plane maps with minimum degree 5, Discrete Math. 313 (2013) 1710-1714. doi:10.1016/j.disc.2013.04.025

[6] O.V. Borodin, A.O. Ivanova and A.V. Kostochka, Every 3-polytope with minimum degree 5 has a 6-cycle with maximum degree at most 11, Discrete Math. 315-316 (2014) 128-134.

doi:10.1016/j.disc.2013.10.021

[7] O.V. Borodin, A.O. Ivanova and D.R. Woodall, Light $C_{4}$ and $C_{5}$ in 3-polytopes with minimum degree 5, Discrete Math. 334 (2014) 63-69. doi:10.1016/j.disc.2014.06.024

[8] O.V. Borodin, A.O. Ivanova and T.R. Jensen, 5-stars of low weight in normal plane maps with minimum degree 5, Discuss. Math. Graph Theory 34 (2014) 539-546. doi:10.7151/dmgt.1748

[9] O.V. Borodin and A.O. Ivanova, Every 3-polytope with minimum degree 5 has a 7-cycle with maximum degree at most 15, Sibirsk. Mat. Zh. 56 (2015) 775-789, in Russian.

[10] B. Ferencová and T. Madaras, On the structure of polyhedral graphs with prescribed edge and dual edge weight, Acta Univ. M. Belii Ser. Math. 12 (2005) 13-18.

[11] B. Ferencová and T. Madaras, Light graph in families of polyhedral graphs with prescribed minimum degree, face size, edge and dual edge weight, Discrete Math. 310 (2010) 1661-1675.

doi:10.1016/j.disc.2009.11.027

[12] Ph. Franklin, The four-color problem, Amer. J. Math. 44 (1922) 225-236. doi:10.2307/2370527

[13] P. Hudák and T. Madaras, On doubly light triangles in plane graphs, Discrete Math. 313 (2013) 1978-1988. doi:10.1016/j.disc.2012.11.018 
[14] S. Jendrol' and T. Madaras, On light subgraphs in plane graphs with minimum degree five, Discuss. Math. Graph Theory 16 (1996) 207-217. doi:10.7151/dmgt.1035

[15] S. Jendrol', T. Madaras, R. Soták and Zs. Tuza, On light cycles in plane triangulations, Discrete Math. 197-198 (1999) 453-467. doi:10.1016/S0012-365X(98)00254-4

[16] S. Jendrol' and H.-J. Voss, Light subgraphs of graphs embedded in the plane-a survey, Discrete Math. 313 (2013) 406-421. doi:10.1016/j.disc.2012.11.007

[17] H. Lebesgue, Quelques conséquences simples de la formule d'Euler, J. Math. Pures Appl. 19 (1940) 27-43.

[18] T. Madaras and R. Soták, The 10 -cycle $C_{10}$ is light in the family of all plane triangulations with minimum degree five, Tatra Mt. Math. Publ. 18 (1999) 35-56.

[19] T. Madaras, On the structure of plane graphs of minimum face size 5, Discuss. Math. Graph Theory 24 (2004) 403-411. doi:10.7151/dmgt.1239

[20] T. Madaras, Two variations of Franklin's theorem, Tatra Mt. Math. Publ. 36 (2007) 61-70.

[21] T. Madaras, R. Škrekovski and H.-J. Voss, The 7-cycle $C_{7}$ is light in the family of planar graphs with minimum degree 5, Discrete Math. 307 (2007) 1430-1435. doi:10.1016/j.disc.2005.11.080

[22] E. Steinitz, Polyeder und Raumeinteilungen, Enzykl. Math. Wiss. 3 (1922) 1-139.

[23] P. Wernicke, Über den kartographischen Vierfarbensatz, Math. Ann. 58 (1904) 413-426. doi:10.1007/BF01444968

Received 22 June 2015

Revised 16 January 2016 Accepted 16 January 2016 